\title{
Basal lamina heparan sulphate proteoglycan is involved in otic placode invagination in chick embryos
}

Accepted: 2 June 2000

\begin{abstract}
Formation of the otocyst from the otic placode appears to differ from invagination of other cup-shaped organ primordia. It is known that the cellular cytoskeleton plays a limited role in otic placode invagination, whilst the extracellular matrix underlying the otic primordium intervenes in the folding process. In this study we have analysed the role of the basal lamina heparan sulphate proteoglycan in otic primordium invagination. At 10 H.H. stage, heparan sulphate proteoglycan immunomarking begins to appear on the otic placode basal lamina, increasing noticeably at 13 H.H. stage, coinciding with maximum folding of the otic epithelium, and is still present at later stages. Enzyme degradation of heparan sulphate proteoglycan in the otic primordium basal lamina, by means of microinjection with heparinase III prior to folding, significantly disrupts invagination of the otic placode, which remains practically flat, with a significant reduction in the depth of the otic pit and an increase in the diameter of the otic opening. The immunocytochemistry analysis revealed a notable depletion of basal lamina heparan sulphate proteoglycan in the otic primordia microinjected with heparinase, with no statistically significant differences observed in the volume or rate of cell proliferation in the otic epithelium relative to the control, which suggests that heparan sulphate proteoglycan disruption does not interfere with the epithelial growth. In addition, a study of apoptosis distribution by the TUNEL method confirmed that treatment with heparinase does not cause interference with cell survival in
\end{abstract}

J.A. Moro-Balbás · A. Gato

Instituto de Neurociencias de Castilla y León, Facultad de Medicina,

Valladolid, Spain

e-mail: moro@med.uva.es

M.I. Alonso · P. Martín · A. de la Mano

Departamento de Anatomía, Facultad de Medicina,Valladolid, Spain

J.A. Moro-Balbás (ه)

Departamento de Anatomía Humana,

Facultad de Medicina, Universidad de Valladolid,

C/ Ramón y Cajal 7, 47005-Valladolid, Spain the otic epithelium. Our findings support the theory that otic primordium invagination may be regulated, at least in part, by the basal lamina components, which might contribute towards anchoring the otic epithelium to adjacent structures.

Key words Otic development $\cdot$ Heparinase . Microinjection · Epithelial folding · Extracellular matrix

\section{Introduction}

The otic placode is an epithelial thickening derived from the surface ectoderm and located close to the hindbrain. Invagination to form the otic pit coincides with the formation of several epithelial folds (Meier 1978; Hilfer et al. 1989). In chick embryos invagination starts at 12 H.H. stage and ends at 16-17 H.H. stage with closure of the otic pit (Álvarez and Navascues 1990). The mechanisms controlling otic placode invagination remain unclear. It has been postulated that the cellular cytoskeleton plays a role in the folding of numerous embryonal epithelial primordia (Hilfer and Searls 1986), and changes in the organisation of actin and myosin could bring about constriction of cell apices, inducing epithelial invagination. In this regard experimental manipulation of ATP levels or disruption in the transport of $\mathrm{Ca}^{++}$, conditions that interfere with the interaction of cytoskeletal actin and myosin, have been shown to disrupt invagination of the thyroid placode (Hilfer et al. 1977), optic vesicle (Brady and Hilfer 1982), nasal placode (Smuts 1981) and neural plate (Lee et al. 1983; Schoenwolf et al. 1988; Ferreira and Hilfer 1993). Nevertheless, these manipulations apparently have no effect on invagination of the otic placode (Hilfer et al. 1989), which indicates that the factors in question do not play a decisive role in the epithelial folds leading to otic vesicle formation.

Extracellular factors, or, more specifically, diverse molecules of the extracellular matrix, have also been associated with epithelial invagination. In fact, it is known that synthesis disruption or enzyme degradation of a cer- 
tain number of these molecules such as collagen, certain proteoglycans and glycoproteins may interfere with epithelial folding, as occurs in branching of the salivary glands, lung and kidney primordia (Klein et al. 1989; Nakanishi et al. 1986; Spooner and Faubion 1980; Spooner et al. 1985; Thompson and Spooner 1983), as well as in the invagination of the otic primordium (Gerchman et al. 1991). It has been demonstrated that treatment with tunicamycin, a drug which inhibits $N$-linked glycosylation, diminishing the synthesis of glycosaminoglycans, disrupts otic primordium invagination (Yang and Hilfer 1982; Rausch and Hilfer 1988). More recently, Gerchman et al. (1995) have shown that the destructuring of the extracellular matrix beneath the otic primordium, by means of treatment with $\beta$-D-xyloside or by enzyme digestion with testicular or streptomyces hyaluronidase, significantly disrupts otic placode invagination due to alteration of the chondroitin sulphate proteoglycan in the extracellular matrix.

The composition and structuring of the basal lamina seems to play an important role in otic placode invagination. There is evidence that attachment of the otic epithelium to the neural tube is required for normal invagination; the otic placode became attached to the neural ectoderm through a single layer of fibronectin and collagen IV between layers of laminin (Hilfer and Randolph 1993) and microinjection of antibodies against laminin detach the two primordia, interfering with invagination of the otic placode (Gerchman et al. 1995). All this appears to indicate that otic placode invagination depends to a large extent on the composition and proper structuring of the extracellular matrix and basal lamina.

Other proteoglycans such as heparan sulphate are present in the basal laminae of embryonal primordia that undergo invagination (Toole 1991), and could play a role in epithelial folding, in this way, it is known that experimental disruption of heparan sulphate proteoglycan (HSPG) alters neural fold elevation during neurulation (Tukett and Morriss-kay 1989) and inhibits branching morphogenesis in embryonic lung explants (Toriyama et al. 1997). Apparently HSPG is involved in the integration of the different components of basal lamina in processes relating to epithelial intercellular adhesion and in establishing epithelium-mesenchyme relations (Jalkanen et al. 1991; Wight et al. 1991; Paulsson 1992; Salmivirta and Jalkanen 1995). Since, as we mentioned above, HSPG is involved in diverse types of epithelial folding during embryonal development, we have tried in this study to determine whether this proteoglycan plays a role in otic placode invagination. We have determined via immunofluorescence the expression of HSPG in the basal lamina of the otic placode between stages 10 to 16 H.H., a period comprising placode invagination. We have also demonstrated its involvement in the folding of the otic epithelium, given that the specific enzyme degradation of this proteoglycan disrupts otic placode invagination.

It is known that HSPG can bind to several growth factors, so this might play a role in regulating the proliferation of cells (Jaakkola and Jalkanen 1999). In this regard we have assessed epithelial volume, the incorporation rate of BrdU and localisation of apoptosis by the TUNEL method in the otic epithelium. Our findings indicate that HSPG enzyme degrading does not appear to interfere with the survival of otic epithelial cells or to have an effect on their growth.

\section{Materials and methods}

For our study we used White Leghorn chick fertile eggs (Ibertec Farms, Valladolid), which were incubated at $38^{\circ} \mathrm{C}$ in a forced draft incubator with $90 \%$ relative humidity.

\section{HSPG-immunomarking}

In order to study the HSPG expression we obtained 10 to $16 \mathrm{H} . \mathrm{H}$. (Hamburger and Hamilton 1951) stage chick embryos. Following extraction the embryos were classified and fixed in Carnoy fluid for $10 \mathrm{~min}$ and, after rapid dehydration in ethanol, embedded in paraplats for $1 \mathrm{~h}$. The embryos were transversally oriented and serially sectioned at $8 \mu \mathrm{m}$. Once rehydrated the sections were washed in TRIS, $\mathrm{pH} 7.4$ buffer, and were treated with testicular hyaluronidase $\left(15,000 \mathrm{U} / \mathrm{ml}\right.$ in TRIS) for $30 \mathrm{~min}$ at $37^{\circ} \mathrm{C}$. After several rinses in TRIS, the sections were incubated in $1 \%$ TRIS/BSA for $20 \mathrm{~min}$ so as to block the possible union of the primary antibody with non-specific proteins. Incubation followed with the first antibody, Rat Ig-G anti-heparan sulphate proteoglycan (Upstate Biotechnology), in a solution of $10 \mu \mathrm{g} / \mathrm{ml}$ in $0.1 \%$ TRIS-BSA for $1 \mathrm{~h}$ at $37^{\circ} \mathrm{C}$. After several rinses in TRIS buffer, the sections were incubated in the secondary antibody, a 1/320 dilution of anti-rat Ig-G FITC conjugate (Sigma), for $1 \mathrm{~h}$ at $37^{\circ} \mathrm{C}$. Following several rinses with TRIS buffer, the sections were mounted in an aqueous medium (Aquamount). For visualisation and photographing of the preparations we used a confocal microscope (Zeiss LSM-310). Control sections were prepared as described above but using pre-immune serum as the primary antibody.

Heparinase microinjection

Microinjecting was carried out "in ovo", subsequent to the opening of a small window in the egg shell. A China ink Ringer solution was subgerminally injected in order to improve visualisation of the embryo, which was then classified by age. Only 9 and 10 H.H. stages embryos were microinjected. Once the vitelline membrane had been cut with tungsten needles, subectodermal microinjection was undertaken, with $2 \mathrm{nl}$ of a solution of $4 \mathrm{U}$ heparinase III from Flavobacterium heparinum (Sigma) dissolved in $5 \mu$ l Ringer, on the right side of the embryo lateral to the hindbrain. On the left side, used as the control, the same amount of heat-deactivated enzyme was microinjected. Microinjection was effected with $10-\mu \mathrm{m}$ tip diameter microneedles connected to a microinjector (Medical Systems, Greenvale, N.Y. 11548, PLI-100). When microinjection had been completed, the egg shell was sealed with a plastic adhesive tape and incubate resumed for a period of $9 \mathrm{~h}$; then, microinjection in the same conditions was repeated, and once again re-incubation took place for a further $9 \mathrm{~h}$, until the embryos reached stage $15-16$ H.H.

A total of 112 embryos were microinjected and, in all of them, the effect produced by treatment with heparinase was checked by direct visualisation and comparison with the treated side (right) relative to the control side (left), and the width of the otic pit opening was noted.

The microinjected embryos were divided in different series for subsequent processing:

1. HSPG-immunomarking, following the same method as the one described above

2. Light microscopy and morphometric analysis 


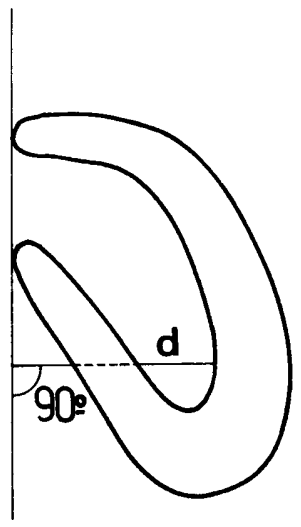

A

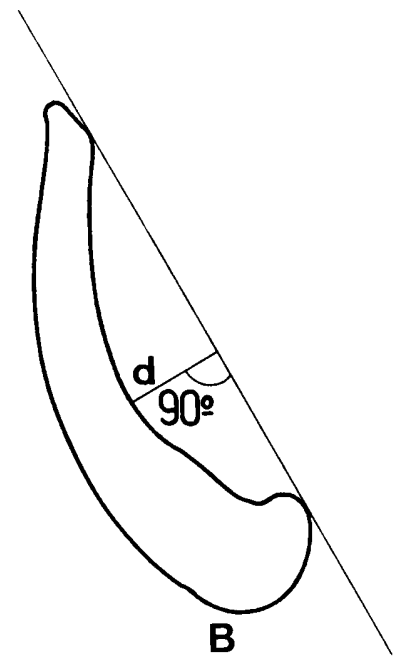

Fig. 1A,B Schematic drawing to show how we measured the depth of the otic pit. First of all we traced a tangent to the edges of the otic pit opening and then another line perpendicular to this up to the deepest area of the otic epithelium; we considered this distance $(d)$ the maximum depth of the otic pit. A Left otic placode (control). B Right otic placode (treated with heparinase III)

3. Apoptosis study by the TUNEL method

4. Study of cell proliferation by BrdU incorporation

5. Scanning electron microscopy

\section{Light microscopy and morphometric analysis}

After microinjection the embryos were extracted from the egg, rinsed in a $37^{\circ} \mathrm{C}$ Ringer solution and the amniotic membrane removed; this completed, they were fixed for a period of $6 \mathrm{~h}$ Bouin's fluid at room temperature. Following dehydration by immersion in graded ethanol, the embryos were embedded in paraplast and 8 - $\mu$ m-transversal sections were obtained; these were processed for staining with haematoxylin-eosin. The most representative sections were photographed with a Nikon microphot-FXA photomacroscope.

In the histological sections we made three types of measurements to assess the degree of growth and epithelial invagination:

1. Calculation of otic epithelium volume

2. Measurement of otic pit depth

3. Measurement of the width of the otic pit opening

In total, 8 embryos were used in these operations

In order to calculate the epithelial volume of the otic primordia, the inner and outer limits of the otic epithelium were drawn in all sections from 16 H.H. stage embryos, by means of a Leitz SM Lux microscope equipped with a drawing tube (total magnification $\times 250$ ). The epithelial areas were measured with a Videoplan (Kontrol Elektronic) computerised image analysis system, with the VIDAS 2.1 sterology program application. With a view to obtaining the total volume of each otic primordium, the areas (expressed in $\mu \mathrm{m}^{2}$ ) were added and multiplied by $8 \mu \mathrm{m}$ (the thickness of the sections). The final volumes were expressed as the arithmetic average of the values obtained \pm standard error.

The diameter of the otic pit opening was measured craniocaudally. From the values obtained the arithmetic mean was calculated and the final results were expressed in $\mu \mathrm{m} \pm$ standard error.

The depth of the otic pit was always measured in the same area, in the middle of the otic primordium; at this level we traced a tangent to the edges of the otic pit opening, then another line perpendicular to the previous one as far as the deepest area of the otic epithelium (Fig. 1). We considered this distance the maximum depth of the otic pit. From the values obtained the arithme- tic mean was calculated and the final results expressed in $\mu \mathrm{m} \pm$ standard error.

To assess the possible differences between the otic primordia treated with heparinase relative to the controls, in all measurements a two-tailed Student $t$-test for independent samples was applied and $P$ values below 0.01 were interpreted as indicating statistical significance.

\section{TUNEL method}

We used this technique to determine the possible effects of the enzyme-degrading of heparan sulphate proteoglycan on the survival of otic epithelium cells whilst assessing apoptosis. The microinjected embryos were fixed in $4 \%$ formaldehyde-PBS and were embedded in paraplast and later sectioned at $8 \mu \mathrm{m}$. To localise apoptosis we employed an Apoptosis Detection System, Fluorescein Kit (Promega) according to the manufacturer's recommendations. This system measures the fragmented DNA of apoptotic cells by catalytically incorporating fluorescein-12-dUTP at the 3'-OH DNA ends using the enzyme terminal deoxynucleotidyl transferase, which forms a polymeric tail using the principle of the TUNEL (Tdt-mediated dUTP Nick-End-Labeling) assay. For visualisation and photographing of the preparations we used a confocal microscope (ZEISS LSM-310).

\section{Cell proliferation assay}

In order to determine whether HSPG enzyme-degrading interferes with cell proliferation in the otic epithelium, we made a study of BrdU incorporation. Following treatment with heparinase III and subsequent re-incubation of the embryos, we administered $20 \mathrm{nl}$ of a $3 \%$ solution of BrdU (Sigma) in PBS by intracardiac microinjection. One $\mathrm{h}$ later the embryos were fixed in Carnoy and embedded in paraplast. Eight- $\mu \mathrm{m}$ sections were deparaffinized, rehydrated in descending series of ethanol, and the endogenous peroxidase blocked by treating the sections with $0.5 \%$ hydrogen peroxide in methanol for $30 \mathrm{~min}$. After several rinses in PBS, the sections were incubated in $4 \mathrm{M} \mathrm{HCl}$ at $37^{\circ} \mathrm{C}$ for $15 \mathrm{~min} . \mathrm{HCl}$ was neutralized by several washes with $0.1 \mathrm{M}$ sodium borate buffer, $\mathrm{pH}$ 8.5. After being rinsed with PBS, the sections were incubated with $6 \mu \mathrm{g} / \mathrm{ml}$ of anti-BrdU antibody (Dako) diluted in PBS at $4{ }^{\circ} \mathrm{C}$ overnight. Detection of this antibody was carried out by means an Antimouse Kit Extravidin Peroxidase (Sigma) according to the manufacturer's instructions. A slight countercolouring with Harris Haematoxylin was performed.

In order to quantify the degree of BrdU incorporation in the otic epithelium cells we counted the number of stained nuclei in the otic pit in alternative sections over a surface of $2,885 \mu \mathrm{m}^{2}$ in each cut; this surface was random in the different sections. We used a total of 6 embryos and the results were expressed as the mean of stained nuclei per surface \pm standard error. To assess whether differences exist in cell proliferation between otic primordia treated with heparinase relative to the controls a twotailed Studen's $t$-test for independent samples was applied and $P$ values below 0.01 were interpreted as indicating statistical significance.

\section{Scanning electron microscopy}

Upon extraction the embryos underwent careful rinsing in a $37^{\circ} \mathrm{C}$ Ringer solution and the amniotic membrane was removed. Following fixing in $2.5 \%$ glutaraldehyde in 0.1 cacodylate buffer, the embryos were dehydrated in acetone. Subsequently, the embryos underwent critical point drying with liquid $\mathrm{CO}_{2}$ in a Balzer's CPD device. Once the embryos had been dried, they were mounted on a metal pedestal and covered with gold film with a Balzer's SCD310 system. A Jeol T-300 scanning electron microscope was used for visualising and photographing the samples. 

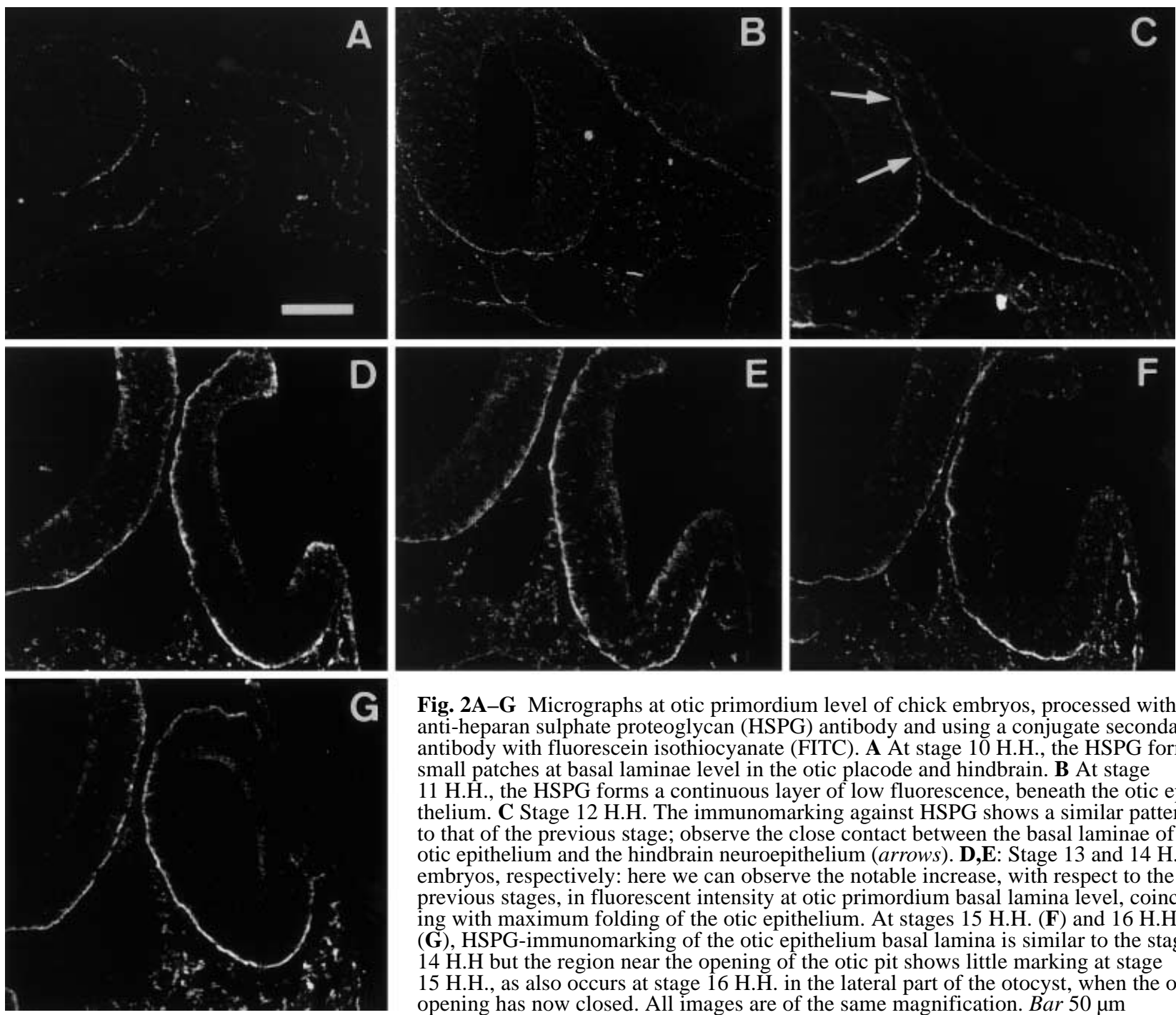

Fig. 2A-G Micrographs at otic primordium level of chick embryos, processed with anti-heparan sulphate proteoglycan (HSPG) antibody and using a conjugate secondary antibody with fluorescein isothiocyanate (FITC). A At stage 10 H.H., the HSPG forms small patches at basal laminae level in the otic placode and hindbrain. B At stage 11 H.H., the HSPG forms a continuous layer of low fluorescence, beneath the otic epithelium. C Stage 12 H.H. The immunomarking against HSPG shows a similar pattern to that of the previous stage; observe the close contact between the basal laminae of the otic epithelium and the hindbrain neuroepithelium (arrows). D,E: Stage 13 and 14 H.H. embryos, respectively: here we can observe the notable increase, with respect to the previous stages, in fluorescent intensity at otic primordium basal lamina level, coinciding with maximum folding of the otic epithelium. At stages 15 H.H. (F) and 16 H.H.

(G), HSPG-immunomarking of the otic epithelium basal lamina is similar to the stage

14 H.H but the region near the opening of the otic pit shows little marking at stage

15 H.H., as also occurs at stage 16 H.H. in the lateral part of the otocyst, when the otic opening has now closed. All images are of the same magnification. Bar $50 \mu \mathrm{m}$

\section{Results}

\section{HSPG expression during otic placode invagination}

We have studied the temporal evolution of otic placode HSPG expression by indirect immunofluorescence between stages 10 and 16 H.H., a period during which invagination of the otic primordium takes place, with the subsequent formation of the otic vesicle.

At 10 H.H. stage the otic epithelium, situated laterally to the hindbrain, begins to thicken, and like the rest of the surface ectoderm, shows hardly any HPGS-immunomarking on the basal lamina; only a few discontinuous patches of low fluorescence can be seen. Slight signs of immunomarking are also visible on the basal lamina of the hindbrain neuroepithelium (Fig. 2A).

At 11 and 12 H.H. stages (Fig. 2B,C), this thickening of the otic epithelium is more pronounced than during the previous stage, but there are still no evident signs of invagination. On the otic placode basal lamina a continuous HSPG layer appears, becoming more fluorescent at stage 12 H.H. At this stage the basal lamina of the hindbrain neuroepithelium shows an immunomarking pattern similar to that of the otic primordium; its dorso-lateral portion is in close contact with the medial part of the otic placode and both basal laminae are attached (Fig. 2C).

During 13 and 14 stages (Fig. 2D,E) a greater change is seen in the shape of the otic placode as a result of the folding of the epithelial wall, which leads to its sinking into the underlying mesenchyme. This epithelial folding is more accentuated in the lateral portion of the otic placode, whilst the medial part undergoes hardly any folding and becomes located very close to the dorso-lateral wall of the hindbrain. At these stages and in coincidence with otic primordium invagination there is a significant increase, in respect of previous stages, in HSPG-immunomarking intensity on the basal lamina of the otic pit. The medial part of the otic placode is still very close to the hindbrain neuroepithelium, but HSPG-immunomarking reveals that its basal laminae are independent, in contrast with stage $12 \mathrm{H}$.H. 

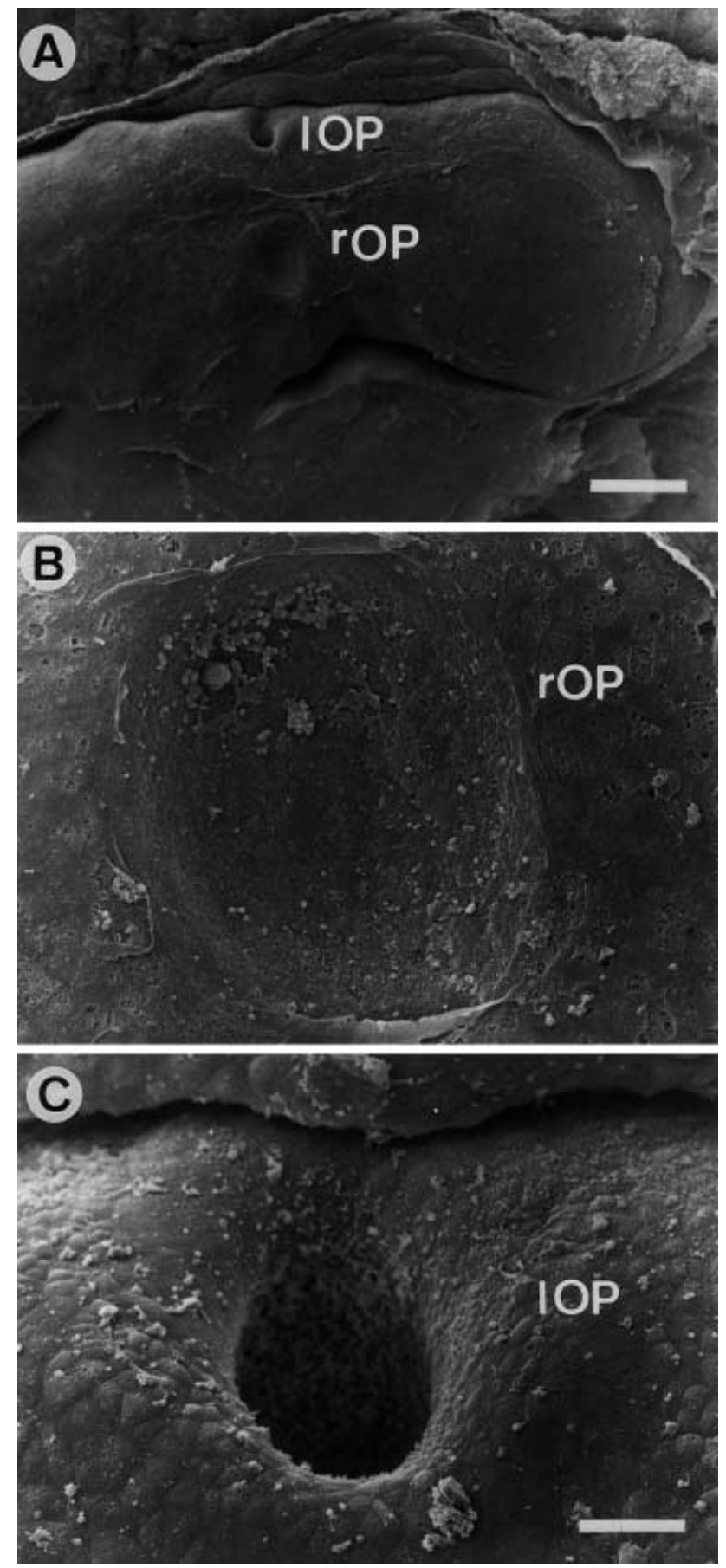

Fig. 3. A Scanning electron micrographs showing the dorsolateral surface of the cephalic extremity of a stage 15 H.H. chick embryo. The right otic placode ( $r O P$ ) has been treated with heparinase III; observe the small degree of invagination with respect to the left, control otic placode (IOP), treated with the same heat de-activated enzyme. Bar $200 \mu \mathrm{m}$. B,C Amplification of the image shown in A, corresponding to the right and left otic primordia, respectively. Bar $25 \mu \mathrm{m}$

At stage 15 H.H. otic epithelium invagination continues, principally at the expense of folding of the most medial portion. The edges of the otic pit come closer together but there is still no contact between them, whilst the otic primordium cavity remains in communication with the amniotic cavity via a small pore (Fig. 2 F). The HSPG-immunomarking pattern is similar to that of previous stages (13 and 14 H.H.), although there is less intense fluorescence in the otic epithelium basal lamina in regions close to the opening of the otic pit (Fig. 2F).

At stage $16 \mathrm{H} . \mathrm{H}$. the edges of the otic pit have established contact and fused to form the otic vesicle, although the latter is still joined to the surface ectoderm (Fig. 2G). The antibody anti-HSPG marks a continuous layer on the basal lamina of the otocyst with an intensity of fluorescence similar to that of the previous stage $(15$ H.H.), with the exception of the lateral part of the otic epithelium, which stays joined to the ectoderm (Fig. 2G).

The effect of treatment with heparinase III on otic placode invagination

In order to determine whether the HSPG present in the otic placode basal lamina plays a role in its invagination, we carried out enzymatic degradation by microinjecting heparinase III into the mesenchyme underlying the right otic primordium at stages 9 to 10 H.H. chick embryos. The heat-inactivated enzyme was microinjected in the left otic primordium, which was used as the control.

Superficially, in almost all embryos the otic pit opening was wider on the side microinjected with heparinase III (right) than on the control side (left), and in the more developed embryos (stage 16 H.H.), the otic opening had closed on this side.

In embryos processed for scanning electron microscopy (Fig. 3), it can be observed how on the right side, microinjected with heparinase III, the otic primordium has undergone hardly any invagination and remains practically flat with a large opening of communication with the exterior, as opposed to the left (control) side, in which invagination has been normal and the otic placode has sunk, despite there being a small opening communicating with the amniotic cavity. The remainder of the embryo appears morphologically normal on both sides. This shows that alterations induced by treatment with heparinase are restricted to the vicinity of the microinjected area.

Light microscopy images (Fig. 5) reveal the same defect with regard to invagination of the right otic placode (treated with heparinase III), and folding of the whole of the medial half is seen to be non-existent; in this area the otic epithelium occasionally appears to have undergone a slight de-structuring, losing the columnar orientation of its nuclei (Fig. 5C), with respect to the left (control) otic anlage (Fig. 5A), which appears pseudo-stratified. Furthermore, the hindbrain neuroepithelium and the medial portion of the otic epithelium are more separated on the side microinjected with heparinase III (right) than on the control side (left; Fig. 5A,C). From a histological point of view, the lateral portion on the right side (the one treated with heparinase III) of the otic epithelium is normal, similar to that of the control side (left), with the cell 

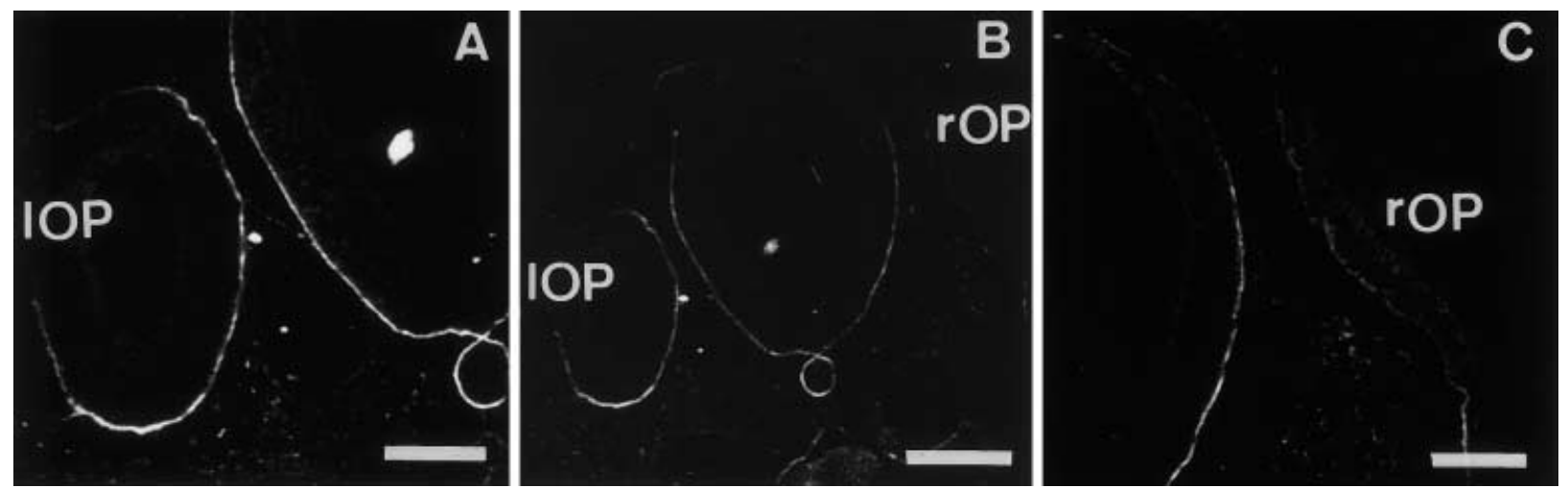

Fig. 4A-C HSPG-immunomarking of a transversal section of a stage 16 H.H. embryo at otic primordium level, showing the effectiveness of enzyme treatment. B The right otic placode $(r O P)$, microinjected with heparinase III has not undergone invagination, and its basal lamina shows little immunofluorescence compared with the control side (1OP), for which invagination has been normal. A,C Amplification of the right and left otic primordia presented in B. Bars B $100 \mu \mathrm{m}$; A,C $50 \mu \mathrm{m}$

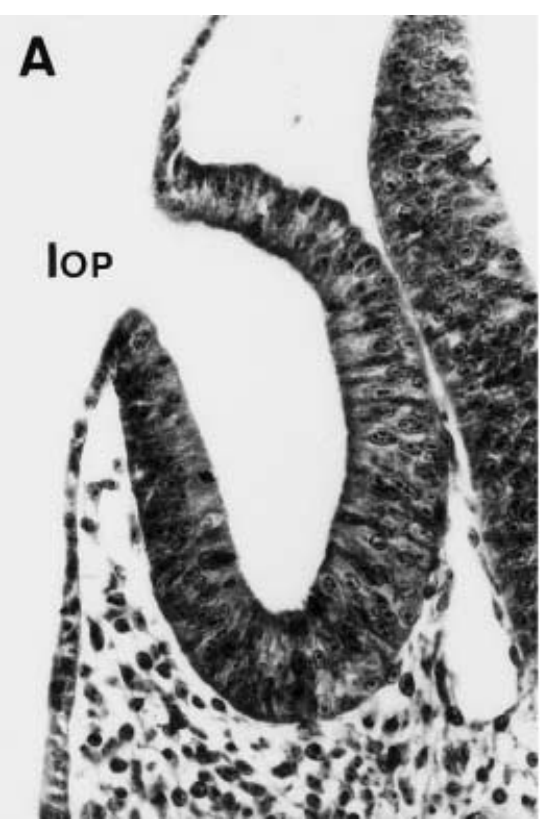

Fig. 5A-C Transversal section of a stage 15 H.H. embryo at otic primordium level. B We can easily observe a flattening with absolutely no invagination at the right otic primordium (rOP) level, as compared with the left, control otic primordium (lOP), which has undergone normal invagination and retains a small pore in communication with the amniotic cavity. A,C amplification of the
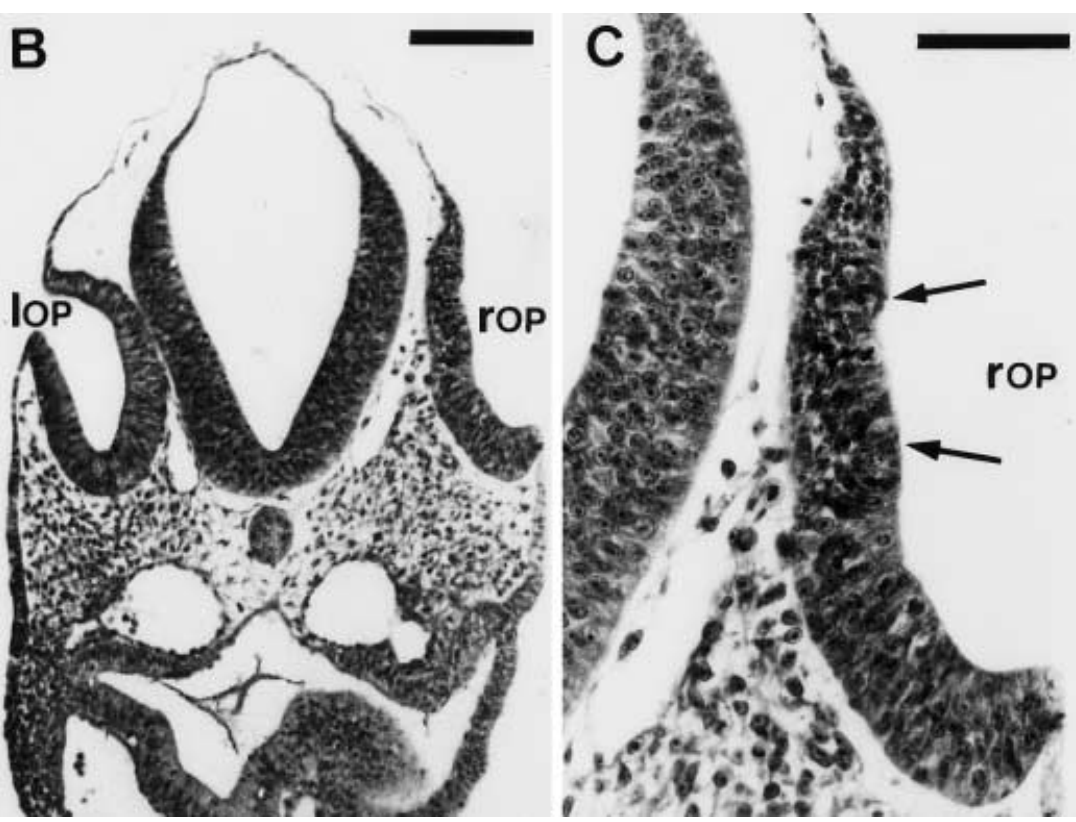

nuclei on both sides arranged in a characteristically pseudo-stratified structure (Fig. 5A,C). These findings indicate that enzymatic degradation of basal lamina HSPG affects the whole process of otic placode invagination, although this influence appears to be more evident in the medial region of the otic pit (next to the hindbrain neuroepithelium).

Alterations in otic placode invagination brought about by treatment with heparinase III are accompanied by acute changes in the HSPG distribution pattern in the basal lamina. Figure 4 shows the effectiveness of enzymatic digestion after $18 \mathrm{~h}$ of treatment. The right right and left otic primordia shown in $\mathbf{B}$. A slight de-structuring of the epithelium in its medial portion (arrow) can be observed in right otic placode $(\mathbf{C})$. In the control side (A) the otic epithelium appears morphologically normal, with its characteristic pseudostratified structure. Bars B $100 \mu \mathrm{m} ; \mathbf{A}, \mathbf{C} 50 \mu \mathrm{m}$

otic placode (treated with heparinase III) has not undergone invagination and a notable reduction can be observed in HSPG-immunomarking on its basal lamina (Fig. 4C) with respect to the left (control) otic primordium, which has now closed so as to form the otic vesicle. This side has an intense positive fluorescent HSPG-band which traces the basal lamina of the otocyst (Fig. 3A), except in the closure region of the otic pit, which remains in contact with the surface ectoderm, this immunomarking pattern corresponds to the one already mentioned for the normal otic primordium in 16 H.H. stage embryos. 
Table 1 Comparison of epithelial volume, otic pit depth, diameter of the otic pit opening and cell proliferation, between otic primordia treated with heparinase III (right otic anlage) and the controls (left otic anlage). Values are given as mean \pm standard error. A two-tailed Student's $t$-test for independent samples was applied and $P$ values below 0.01 were interpreted as indicating statistical significance

\begin{tabular}{|c|c|c|c|c|}
\hline & $\begin{array}{l}\text { Epithelial volume } \\
\left(\mu \mathrm{m}^{3} \times 10^{3}\right)\end{array}$ & $\begin{array}{l}\text { Depth of the otic pit } \\
(\mu \mathrm{m})\end{array}$ & $\begin{array}{l}\text { Opening of the otic } \\
\text { pit }(\mu \mathrm{m})\end{array}$ & $\begin{array}{l}\text { BrdU-labeled } \\
\text { cells/area }\end{array}$ \\
\hline Right otic anlage & $1730.12 \pm 97.04$ & $40.93 \pm 10.09 *$ & $237.33 \pm 34.36^{*}$ & $25.3 \pm 6$ \\
\hline Left otic anlage & $\begin{array}{l}1720.83 \pm 99.69 \\
(n=8)\end{array}$ & $\begin{array}{l}87.66 \pm 12.22 * \\
(n=8)\end{array}$ & $\begin{array}{l}56 \pm 36.48 * \\
(n=8)\end{array}$ & $\begin{array}{l}27.7 \pm 2.45 \\
(n=6)\end{array}$ \\
\hline
\end{tabular}

\footnotetext{
$* P<0.01$

a Area $=2885 \mu \mathrm{m}^{2}$
}

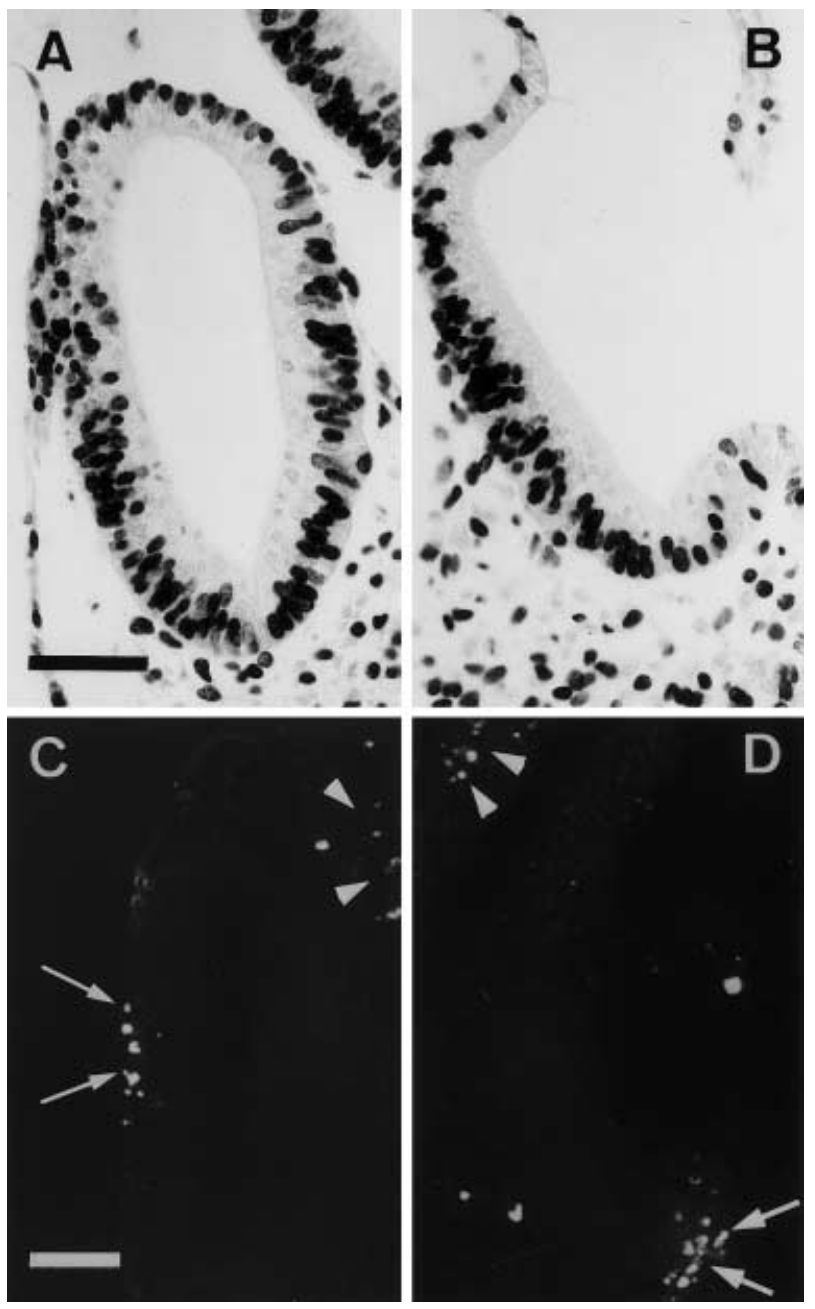

Fig. 6 BrdU immunolabelling $(\mathbf{A}, \mathbf{B})$ and apoptosis detected by the TUNEL method $(\mathbf{C}, \mathbf{D})$ in stage 16 H.H. chick embryos. A Otic vesicle of the left side (control). B Right otic placode treated with heparinase III, showing a clear delay in invagination relative to the left side. On both sides staining intensity and the number of nuclei stained with BrdU is similar, and cells in the DNA synthesis phase are located mainly at the basal portion of the otic epithelium. Apoptosis on the control side $(\mathbf{C})$ was only observed in the region of closure and fusion of the otic opening (arrows). On the side treated with heparinase III (D) the otic placode has barely undergone invagination and apoptosis is present solely on the lateral edge of the otic pit (arrows). Occasionally we found isolated apoptosis in the hindbrain neuroepithelium (arrowheads) on both sides near to the otic pit. Bars $50 \mu \mathrm{m}$
Morphometric analysis

In order to determine whether treatment with heparinase affects otic epithelium growth and so as to evaluate the extent of interference in otic placode invagination induced by degrading the HSPG in the basal lamina, we made three different measurements in the histological sections: (1) Calculation of the volume of the otic epithelium, (2) Measurement of the depth of the otic pit, (3) Measurement of the diameter of the otic pit opening.

In all the measurings, and in order to compare the treated side with that of the control, a two-tailed Student's $t$-test for independent samples was applied and $P$ values below 0.01 were interpreted as indicating statistical significance. The values obtained are summarised in Table 1.

Comparison of the epithelial volumes of the otic primordia treated with heparinase III and the controls revealed no statistically significant variations $(P>0.01)$. This suggests that degrading the basal lamina HSPG does not interfere with otic epithelium growth. However, the depth of the otic pit was more than double on the control side, whilst the diameter of the otic pit opening was considerably larger (four times) on the side treated with heparinase III. Both measurements reflected statistically significant differences $(P<0.01)$ in the degree of invagination between the right otic pit (treated side) and the left (control side). This shows that enzyme degrading of the basal lamina HSPG clearly interferes with otic placode invagination.

\section{Study of apoptosis and cell proliferation}

In order to check whether enzyme degrading of basal lamina HSPG interferes with cell survival in the otic epithelium, we analysed at this level the presence of apoptosis by the TUNEL method. On the control side (left) apoptosis was only observed on the edges of the otic opening, and coincided with the closure and fusion and subsequent isolation of the otic pit from the surface ectoderm in the formation of the otic vesicle (Fig. 6C). As was to be expected, on the side treated with heparinase III (right) there was a considerable delay in otic pit invagination and only one area of apoptosis could be observed on the lateral edge of the otic pit opening, in the transition zone between the otic epithelium and the ce- 
phalic ectoderm (Fig. 6D). Apart from in these areas, which can be considered physiological or ones in which cellular death is normal, we found no sign of apoptosis in the rest of the otic epithelium. We also encountered several isolated cell deaths in the dorsolateral wall of the hindbrain neuroepithelium on both the control side and that treated with heparinase III (Fig. 6C,D).

By studying BrdU incorporation, we found that most cells in the DNA synthesis phase were located near to the basal portion of the otic epithelium and that there were few cells stained with BrdU in the vicinity of the apical portion on both the side treated with heparinase III and the control side (Fig. 6A,B); what is more, staining intensity on both sides was similar. In order to check whether HSPG enzyme degrading interferes with the proliferation of cells in the otic epithelium, we counted the cells stained with BrdU per surface unit (Table 1). We observed that there was a slight decrease in BrdU incorporation on the side treated with heparinase relative to the control side; however, the statistical study revealed no statistically significant differences between the two sides $(P>0.01)$, which suggests that the disruption of basal lamina HSPG does not interfere with cell proliferation in the otic epithelium.

\section{Discussion}

The basal lamina of the otic placode during invagination has a complex structure, and the presence of different components such as laminin, fibronectin, collagen IV and cytotactin has been described (Richardson et al. 1987; Hilfer and Randolph 1993). Both qualitative and quantitative differences exist in the distribution of these molecules in the otic placode. Cytotactin tends to be located in the lateral portion, whilst the medial part of the otic primordium comes into contact with the neural tube neuroepithelium; at this level the basal laminae of both epithelia are attached by thin layers of laminin, collagen IV and fibronectin (Hilfer and Randolph 1993) and there is data that indicates that attachment of the otic epithelium to the neural tube is required for normal invagination. In this regard it has been demonstrated that microinjection of antibodies against laminin detach the two primordia, interfering with otic placode invagination (Gerchman et al. 1995). In our study we have shown that the HSPG forms part of the otic placode basal lamina, and we have demonstrated that there is a progressive increase in the concentration of this proteoglycan from stages 10 to $13 \mathrm{H}$.H., when maximum folding of the otic epithelium occurs (Álvarez and Navascues 1990) and there is also close contact between the basal laminae of the medial part of the otic pit and the neural tube. This appears to be related to otic placode invagination, since basal lamina HSPG degradation by microinjection with heparinase III alters otic epithelium folding. The absence of Ac. anti-heparan sulphate immunomarking on the basal lamina of the treated otic primordium shows the effectiveness of treatment with heparinase.
The integrity of the extracellular matrix and the basal lamina seems necessary for normal morphogenesis of the inner ear, and certain studies support the thesis that glycoproteins may play an important role in otic placode invagination. Treatment of the otic primordium with tunicamycin (Rausch and Hilfer 1988), a drug which inhibits $N$ linked glycosylation (Mahoney and Duskin 1979), disrupts placode invagination. Microinjection of $\beta$-D-xyloside in the extracellular matrix beneath the otic placode causes delayed invagination in chick (Gerchman et al. 1995) and rat (Alonso et al. 1998, 1999) embryos, this drug disrupts the synthesis of sulphated proteoglycans, particularly chondroitin sulphate proteoglycan and HSPG (Sobue et al. 1987; Lugemwa and Esko 1991; Lugemwa et al. 1996). It has also been demonstrated that enzymatic degradation of extracellular matrix hyaluronic acid disrupts otic epithelium folding (Gerchman et al. 1995). Previously mentioned data and the present results suggest that glycoprotein molecules and especially proteoglycans play an important role in otic placode invagination, but the mechanism by which these molecules may control epithelial folding is not clear. Hyaluronic acid and sulphate proteoglycans are known to possess great hydrophylic capacity (Compert and Zamparo 1990) and can bring about an increase in intercellular spaces in the extracellular matrix that could exert a mechanical force on the edges of the otic epithelium and facilitate its folding. In this regard we know that hydration caused by hyaluronic acid and proteoglycans is an important factor in neuroepithelium folding in the formation of the neural tube (Schoenwolf and Fisher 1983; Morriss-Kay and Tuckett 1989).

A description has also been given of the presence of adhesion molecules in the otic epithelium during invagination, more specifically N-CAM and L-CAM (Richardson et al. 1987); the former appears throughout the whole of the epithelium but is more concentrated in the lateral portion, whilst L-CAM has a uniform distribution pattern. Recently Brown et al. (1998) demonstrated that in avian embryos microinjection of blocking antibodies to $\mathrm{N}$ CAM into the paraxial mesoderm adjacent to the otic placode resulted in two major classes of defects: detachment of the primordium from the neural tube and interference with formation of the otic folds. It is known that HSPG may interact with N-CAM at plasmatic membrane level (Cole and Burg 1989) and this interaction might produce changes in N-CAM structure that modulate cellular adhesion, thereby facilitating epithelial folding.

It has also been suggested that cell surface proteoglycans might establish connections between the intracellular cytoskeleton and the extracellular matrix (Goetinck 1991; Jalkanen et al. 1991), which could bring about changes in the shape of the cells that would condition the folding of the epithelium. However, as was pointed out in the introduction, the role of the cellular cytoskeleton appears to be of little relevance in otic placode invagination.

HSPG is known to be capable of joining with different components of the extracellular matrix via its chains of glycosaminoglycan (Goetinck 1991). These interactions between HSPG and the extracellular matrix might 
explain the fact that enzymatic digestion of the HSPG inhibits otic placode invagination, and it would constitute a point in common with alterations in otic primordium folding induced by the alteration of other components of the extracellular matrix. The HSPG may join with the fibronectin (Toole 1991; Paulsson 1992), which in turn is capable of joining with the collagen IV (Yamada 1985); these molecules form part of the otic placode basal lamina during its invagination and, in fact, contribute to hindbrain neuroepithelium and otic epithelium anchoring (Hilfer and Randolph 1993). This anchoring apparently plays an important role in epithelial folding, since it could stabilise a part of the epithelium whilst in other regions active or passive movements occur that lead to epithelial invagination. Enzymatic digestion of the HSPG could de-stabilise these connections between the otic epithelium and the neural tube and this might account for the disruption in invagination induced by treatment with heparinase. What is more, we have observed that although disruption of invagination is generalised, it is more visible in the medial part of the otic placode, and that there is separation between otic and neural epithelia. These factors support the hypothesis concerning the importance of otic placode basal lamina HSPG in its union with the hindbrain neuroepithelium.

It has also been postulated that a differential growth of the otic primordium could bring about internal forces within the epithelium leading to its invagination (Álvarez and Navascues 1990), as occurs in the neuroepithelium during elevation of the neural folds (Sausedo et al. 1997) or in lung-branching morphogenesis (Gallagher 1986). In this regard HSPG might stimulate epithelial growth on account of its association with growth factors. It is known that HSPG can bind to several growth factors, modulating its activity (Goetinck 1991; Ruoslahti and Yamaguchi 1991; Aviezer et al. 1994a,b; Johnson and Wong 1994; Schuger et al. 1996; David and Bernfield 1998). This interaction has been studied mainly for fibroblast growth factors (FGFs) and several mechanisms have been proposed (Jaakkola and Jalkanen 1999) to explain how HSPG may modulate the action of the FGFs. First, the glycosaminoglycans in proteoglycans may induce oligomerization of FGF molecules required for fibroblast growth factor receptor clustering. Second, proteoglycans could protect FGFs from proteolytic degradation and serve as a reservoir for these heparin-binding growth factors. Third, HSPG could simply serve as molecules that have an ability to present the FGFs to the high-affinity receptors. Finally, it has been hypothesised that the main function of proteoglycans is to reduce the dimensionality of FGF diffusion. In addition, it has been proven that HSPG enzyme degradation or treatment with heparan sulphate antibody inhibits lung-branching morphogenesis in mouse embryos and that addition of basic fibroblast growth factor - a typical heparin-binding growth factor restored the inhibition caused by heparitinase (Schuger et al. 1996; Toriyama et al. 1997).

As regards otic primordium development, it has been shown that different secreted factors like the BMP-family of TGF $\beta$-like polypeptides, FGF-2 and FGF-3 are expressed in the otic placode and otic vesicle (Oh et al. 1996; Wu and Oh 1996; Mahamood et al. 1995, 1996; Torres and Giráldez 1998). It is not clear whether these factors can have an effect in otic placode invagination, although it has been suggested that FGFs might play a role in this process. This hypothesis was supported by the observation that otocyst formation in chick embryos was blocked by anti-sense oligonucleotides to FGF-3 protein and by antibodies against FGF-3 in explant culture (Represa et al. 1991). However, mice homozygous for a targeted disruption of the FGF-3 gene form otocyts that are normal in morphology but whose subsequent morphogenesis and differentiation are aberrant (Mansour et al. 1993). Similarly, in the Kreisler mutant mouse, in which FGF-3 is expressed at very low levels (Frohman et al. 1993; McKay et al. 1994), the otocyst forms but fails to undergo normal morphogenesis and differentiation (Deol 1964). These studies appear to indicate that FGF-3 is essential for late stages of otic morphogenesis and differentiation but not for invagination of the otic placode.

Since, as we have already mentioned, HSPG may modulate the activity of growth factors that could have an influence on otic primordium development, we have made an analysis of apoptosis, cell proliferation and the size of the otic epithelium. Cellular apoptosis detected by the TUNEL method was observed solely on the lateral edge of the otic pit on the side treated with heparinase III, and in the fusion and closure area of the otic opening on the control side. Both these areas of apoptosis may be considered physiological as they appear during normal development of the otic primordium (Marovitz et al. 1977; Represa et al. 1990). Analysis of otic epithelium size showed no statistically significant differences between the treated and control sides; similarly, although there was a slight reduction in the number of BrdU stained cells on the treated side, this difference was not statistically significant. This suggests that enzymedegrading of basal lamina HSPG does not interfere with the survival or proliferation of cells or with otic epithelium growth; however, we cannot rule out the co-participation of HSPG and growth factors in otic placode invagination, which might act in a different way from that of regulation of cell proliferation.

To conclude, this study provides experimental evidence that the integrity of basal lamina components is a requisite for normal otic placode invagination, despite the fact that we cannot exclude the co-intervention of other factors in otic epithelium folding.

\section{References}

Alonso MI, Gato A, Moro JA, Martín P, Barbosa E (1998) Neural tube defects induced by $\beta$-D-xyloside: evidence of a role for sulfated proteoglycans in neural fold fusion in rat embryos. Eur J Anat 2:133-140

Alonso MI, Gato A, Moro JA, Martín P, Barbosa E (1999) Involvement of sulfated proteoglycans in embryonic brain expansion at earliest stages of development in rat embryos. Cells Tissues Organs 165:1-9 
Álvarez IS, Navascues J (1990) Shaping, invagination, and closure of the chick embryo otic vesicle: scanning electron microscopic and quantitative study. Anat Rec 228:315-326

Aviezer D, Levy E, Safran M, Svahn C, Budderke E, Shmidt E, David G, Vlodavsky I, Yayon A (1994a) Differential structural requirements of heparin and heparan sulfate proteoglycans that promote binding of bFGF to its receptor. J Biol Chem 269: $114-121$

Aviezer D, Hecht D, Safran M, Eisinger M, David G, Yayon A (1994b) Perlecan, basal lamina proteoglycan, promotes basic fibroblast growth factor-receptor binding, mitogenesis. Cell 79: $1005-1013$

Brady RC, Hilfer SR (1982) Optic cup formation: a calcium regulated process. Proc Natl Acad Sci USA 79:5587-5591

Brown JW, Beck-Jefferson E, Hilfer SR (1998) A role for neural cell adhesion molecule in the formation of the avian inner ear. Dev Dyn 213:359-369

Cole GJ, Burg M (1989) Characterization of a heparan sulfate proteoglycan that copurifies with the neural cell adhesion molecule. Exp Cell Res 182:44-60

Compert WD, Zamparo O (1990) Hydrodynamic properties of connective tissue polysaccharides. Biochem J 269:561-564

David G, Bernfield M (1998) The emerging roles of cell surface heparan sulfate proteoglycans. Matrix Biol 17:461-463

Deol MS (1964) The abnormalities of the inner ear Kreisler mice. J Embryol Exp Morphol 11:475-490

Ferreira MC, Hilfer SR (1993) Calcium regulation of neural fold formation; visualization of the actin cytoskeleton in living chick embryos. Dev Biol 427:427-440

Frohman MA, Martin GR, Cordes SP, Halamek L, Barsh GS (1993) Altered rhombomere specific expression and hyoid bone differentiation in the mouse segmentation mutant Kreisler $(\mathrm{Kr})$. Development 117:925-936

Gallagher BC (1986) Branching morphogenesis in the avian lung: electron microscopic studies using cationic dyes. J Embryol Exp Morphol 94:189-205

Gerchman EM, Hilfer SR, Simmons DM (1991) Differential involvement of glycosaminoglycans in organogenesis of the avian inner ear and eye. J Cell Biol 114:293a (abstract)

Gerchman E, Hilfer SR, Brown JW (1995) Involvement of extracellular matrix in the formation of the inner ear. Dev Dyn 202: 421-432

Goetinck PF (1991) Proteoglycans in development. Curr Top Dev Biol 25:111-131

Hamburger V, Hamilton HL (1951) A series of normal stages in the development of the chick embryo. J Morphol 88:49-92

Hilfer SR, Searls RL (1986) Cytoskeletal dynamics in animal morphogenesis. Dev Biol 2:3-29

Hilfer SR, Randolph GJ (1993) Immunolocalization of basal lamina components during development of chick otic and optic primordia. Anat Rec 235:443-452

Hilfer SR, Palmatier BY, Fithian EM (1977) Precocious evagination of the embryonic chick thyroid in ATP-containing medium. J Embryol Exp Morphol 42:163-175

Hilfer SR, Esteves RA, Sanzo JF (1989) Invagination of the otic placode: Normal development and experimental manipulation. J Exp Zool 251:253-264

Jaakkola P, Jalkanen M (1999) Transcriptional regulation of syndecan-1 expression by growth factors. Prog Nucleic Acid Res Mol Biol 63:109-138

Jalkanen M, Jalkanen S, Bernfield M (1991) Binding of extracellular effector molecules by cell surface proteoglycans. In: Mcdonald JA, Mecham RP (eds) Receptors for extracellular matrix. Academic Press, San Diego, pp 1-37

Johnson GR, Wong L (1994) Heparan sulfate is essential to amphiregulin-induced mitogenic signaling by the epidermal growth factor receptor. J Biol Chem 269:27149-27154

Klein DJ, Brown DM, Moran A, Oegema TR Jr, Platt JL (1989) Chondroitin sulfate proteoglycan synthesis and reutilization of $\beta$-D-xyloside-initiated chondroitin/dermatan sulfate glycosaminoglycans in fetal kidney branching morphogenesis. Dev Biol 133:515-528
Lee HY, Kosciuk MC, Nagele RG, Roisen FJ (1983) Studies on the mechanisms of neurulation in the chick: Possible involvement of myosin in elevation of neural folds. J Exp Zool 225: 449-457

Lugemwa FN, Esko JD (1991) Estradiol $\beta$-D-xyloside, an efficient primer for heparan sulfate biosynthesis. J Biol Chem 266: 6674-6677

Lugemwa FN, Sarkar AK, Esko JD (1996) Unusual beta-D-xylosides that prime glycosaminoglycans in animal cells. J Biol Chem 271:19159-19165

Mahamood R, Kiefer P, Gunthrie S, Dickson C, Mason I (1995) Multiple roles for FGF-3 during cranial neural development in the chicken. Development 121:1399-1410

Mahmood R, Mason IJ, Morriss-Kay GM (1996) Expression of FGF-3 in relation to hindbrain segmentation, otic pit position and pharyngeal arch morphology in normal and retinoic acidexposed mouse embryos. Anat Embryol 194:13-22

Mahoney WC, Duskin D (1979) Biological activities of the two major components of tunicamycin. J Biol Chem 254:65726576

Mansour SL, Goddard JM, Capecchi MR (1993) Mice homozygous for a targeted disruption of the proto-oncogene int- 2 have developmental defect in the tail and inner ear. Development 117: $13-28$

Marovitz WF, Khan KM, Schulte T (1977) Ultrastructural development of the early rat otocyst. Ann Otol Rhinol Laryngol 86:9-28

McKay IJ, Muchamore I, Krumlauf R, Maden M, Lumsden A, Lewis J (1994) The Kreisler mouse: a hindbrain segmentation mutant that lacks two rhombomeres. Development 120:2199_ 2211

Meier S (1978) Development of the embryonic chick otic placode. I. Light microscopic analysis. Anat Rec 191:447-458

Morriss-Kay G, Tuckett F (1989) Inmunohistochemical localisation of chondroitin sulfate proteoglycans and the effets of chondroitinase $\mathrm{ABC}$ in 9 to 11 day rat embryos. Development 106: 787-798

Nakanishi Y, Sugiura F, Kishi J, Hayakawa T (1986) Local effects of implanted Elvax chips containing collagenase inhibitor and bacterial collagenase on branching morphogenesis of mouse embryonic submandibular glands in vitro. Zool Sci 3:479-486

Oh SH, Johnson R, Wu DK (1996) Differential expression of bone morphogenetic proteins in the developing vestibular and auditory sensory organs. J Neurosci 16:6463-6475

Paulsson M (1992) Basement membrane proteins: structure, assembly, and cellular interactions. Crit Rev Biochem Mol Biol 27:93-127

Rausch DA, Hilfer SR (1988) Putative role for extracellular matrix in otic vesicle formation. J Cell Biol 107:795a

Represa JJ, Moro JA, Gato A, Pastor F, Barbosa E (1990) Patterns of epithelial cell death during early development of the human inner ear. Ann Otol Rhinol Laryngol 99:482-488

Represa J, León Y, Miner C, Giráldez F (1991) The int-2 proto-oncogene is responsible for induction of the inner ear. Nature 353:561-563

Richardson GP, Corssin KL, Chuong Ch-M, Edelman GM (1987) Expression of cell adhesion molecules during embryonic induction. III. Development of the otic placode. Dev Biol 119: 217-230

Ruoslahti E, Yamaguchi Y (1991) Proteoglycans as modulators of growth factor activities. Cell 64:867-869

Salmivirta M, Jalkanen M (1995) Syndecan family of cell surface proteoglycans: developmentally regulated receptors for extracellular effector molecules. Experientia 51:863-871

Sausedo RA, Smith JL, Schoenwolf GC (1997) Role of nonrandomly oriented cell division in shaping and bending of the neural plate. J Comp Neurol 381:473-488

Schoenwolf GC, Fisher M (1983) Analysis of the effects of streptomyces hyalurinidase on formation of the neural tube. J Embryol Exp Morphol 73:1-15

Schoenwolf GC, Folsom D, Moe A (1988) A reexamination of the role of microfilaments in the chick embryo. Anat Rec 220: 87-102 
Schuger L, Johnson GR, Gilbride K, Plowman GD, Mandel R (1996) Amphiregulin in lung branching morphogenesis: interaction with heparan sulfate proteoglycan modulates cell proliferation. Development 122:1759-1767

Smuts MS (1981) Rapid nasal pit formation in mouse embryos stimulated by ATP-containing medium. J Exp Zool 216: 409-414

Sobue M, Habichi H, Ito K, Yonekura H, Sakurai K, Kamohara S, Ueno Y, Noyori R, Suzuki S (1987) $\beta$-D-xylosides and their analogues as artificial initiators of glycosaminoglycan chain synthesis. Biochem J 24:591-601

Spooner BS, Faubion J (1980) Collagen involvement in branching morphogenesis of embryonic lung and salivary gland. Dev Biol 77:84-102

Spooner BS, Bassett K, Stokes B (1985) Sulfated glycosaminoglycan deposition and processing at the basal epithelial surface in branching and in $\beta$-D-xyloside-inhibited embryonic salivary glands. Dev Biol 109:177-183

Thompson HA, Spooner BS (1983) Proteoglycan and glycosaminoglycan synthesis in embryonic mouse salivary glands: Effects of $\beta$-D-xyloside, an inhibitor of branching morphogenesis. J Cell Biol 96:1443-1450
Toole BP (1991) Proteoglycans and hyaluronan in morphogenesis and differentiation. In: Hay ED (ed) Cell biology of extracellular matrix, 2nd edn. Plenum Press, New York, pp 305-341

Toriyama K, Muramatsu H, Hoshino T, Torii S, Muramatsu T (1997) Evaluation of heparin-binding growth factors in rescuing morphogenesis of heparitinase-treated mouse embryonic lung explants. Differentiation 61:161-167

Torres M, Giráldez F (1998) The development of the vertebrate inner ear. Mech Dev 71:5-21

Tuckett F, Morriss-Kay GM (1989) Heparitinase treatment of rat embryos during cranial neurulation. Anat Embryol 180:393-400

Wight ThN, Heinegard DK, Hascall VC (1991) Proteoglycans: structure and function. In: Hay ED (ed) Cell biology of extracellular Matrix, 2nd edn. Plenum Press, New York, pp 45-78

Wu DK, Oh SH (1996) Sensory organ generation in the chick inner ear. J Neurosci 16:6454-6462

Yamada KM (1985) Cell surface interactions with extracellular materials. Ann Rev Biochem 52:161-199

Yang J-W, Hilfer SR (1982) Interference with ocular development by inhibitors of glycoconjugate synthesis. Dev Biol 92:41-53 